Keywords: angiogenesis; carbohydrate metabolism; endothelial cell; extracellular matrix; glycobiology; hyaluronate; oligosaccharide; tumour interstitial fluid

\title{
Accumulation of small hyaluronan oligosaccharides in tumour interstitial fluid correlates with lymphatic invasion and lymph node metastasis
}

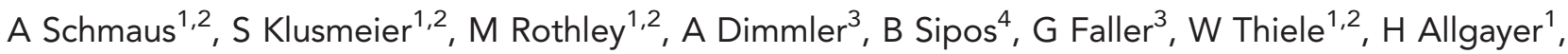 \\ P Hohenberger ${ }^{1}$, S Post ${ }^{1}$ and J P Sleeman ${ }^{*, 1,2}$ \\ ${ }^{1}$ Medical Faculty Mannheim, University of Heidelberg, Ludolf-Krehl-Strasse 13-17, 68167 Mannheim, Germany; ${ }^{2}$ Karlsruhe Institute \\ for Technology (KIT), Campus Nord, Institut für Toxikologie und Genetik, Postfach 3640, 76021 Karlsruhe, Germany; ${ }^{3}$ Institut und \\ Gemeinschaftspraxis für Pathologie an den St Vincentiuskliniken Karlsruhe, Südendstrasse 37, 76137 Karlsruhe, Germany and \\ ${ }^{4}$ Universitätsklinikum Tübingen, Department of Pathology, Liebermeisterstrasse 8, 72076 Tübingen, Germany
}

Background: Association studies have implicated the glycosaminoglycan hyaluronan (hyaluronic acid, HA) and its degrading enzymes the hyaluronidases in tumour progression and metastasis. Oligosaccharides of degraded HA have been ascribed a number of biological functions that are not exerted by high-molecular-weight HA (HMW-HA). However, whether these small HA oligosaccharides (sHA) have a role in tumour progression currently remains uncertain due to an inability to analyse their concentration in tumours.

Methods: We report a novel method to determine the concentration of sHA ranging from 6 to 25 disaccharides in tumour interstitial fluid (TIF). Levels of sHA were measured in TIF from experimental rat tumours and human colorectal tumours.

Results: While the majority of HA in TIF is HMW-HA, concentrations of sHA up to $6 \mu \mathrm{g} \mathrm{ml}^{-1}$ were detected in a subset of tumours, but not in interstitial fluid from healthy tissues. In a cohort of 72 colorectal cancer patients we found that increased sHA concentrations in TIF are associated with lymphatic vessel invasion by tumour cells and the formation of lymph node metastasis.

Conclusions: These data document for the first time the pathophysiological concentration of sHA in tumours, and provide evidence of a role for sHA in tumour progression.

Hyaluronan (hyaluronic acid, HA) is an unbranched polysaccharide composed of up to 25000 disaccharide units of glucuronic acid and $\mathrm{N}$-acetylglucosamine. It is synthesised by three different membrane-bound HA synthases, extruded directly into the extracellular space and comprises an important component of the extracellular matrix (Itano et al, 1999a). In a variety of carcinomas, HA accumulates in the tumour stroma and on the surface of malignant cells. High HA levels correlate with poorly differentiated tumours and shorter patient survival (Ropponen et al, 1998; Setälä et al, 1999; Anttila et al, 2000; Auvinen et al, 2000; Lokeshwar et al, 2001; Pirinen et al, 2001; Posey et al, 2003). HA production by tumours has been suggested to increase proliferation, promote tumour cell invasion and epithelialmesenchymal transition, and stimulate vessel growth and the recruitment of stromal cells (Kosaki et al, 1999; Itano et al, 1999b; Jacobson et al, 2002; Koyama et al, 2007, 2008; Sironen et al, 2011). Nevertheless, accumulation of HA can decrease tumourigenic potential (Zhang et al, 1995; Enegd et al, 2002; Itano et al, 2004; 
Bharadwaj et al, 2009) and confer resistance to cancer (Tian et al, 2013). Moreover, expression of HA-degrading enzymes, the hyaluronidases, also correlates with tumour progression (Posey et al, 2003; Ekici et al, 2004; Stern, 2005; Udabage et al, 2005; Simpson and Lokeshwar, 2008). As the concurrent expression of HA-synthesising and -degrading enzymes in tumours provides the strongest stimulus for tumour growth and metastasis formation (Enegd et al, 2002; Simpson, 2006; Bharadwaj et al, 2009), enhanced turnover of accumulated HA therefore appears to be central to the contribution of HA to these processes.

Physiologically, HA is turned over continuously (Laurent and Fraser, 1992), in a process in which initial intermediate-sized fragments produced extracellularly are internalised and further degraded (Stern, 2003). Extracellular HA degradation is effected by secreted or membrane-bound hyaluronidases, and by reactive oxygen species (Soltés et al, 2006; Stern et al, 2007). Although HA fragments are normally rapidly cleared, increased synthesis and breakdown of HA in the tumour context could potentially lead to the accumulation of small HA oligosaccharides (sHA) in the interstitial fluid (Stern et al, 2007), usually defined as HA of 4-25 disaccharides. In experimental systems, sHA has wide-ranging biological activities that are not exerted by high-molecular-weight HA (HMW-HA; Stern et al, 2006). For example, while HMW-HA is not angiogenic, sHA is a potent inducer of angiogenesis in vitro and in vivo (West et al, 1985; Matou-Nasri et al, 2009; Gao et al, 2010). Furthermore, sHA can promote migration and invasion of tumour cells, for example, by increasing the expression of matrix metalloproteases and cytokines, and by inducing proteolytic cleavage of CD44 (Sugahara et al, 2003; Fieber et al, 2004; Voelcker et al, 2008). Thus, tumour interstitial fluid (TIF) is an appropriate compartment within which to measure pathophysiologically relevant sHA concentrations.

Despite the possible accumulation of sHA in tumours and its potential to affect tumour growth and progression, few studies have investigated the presence of $\mathrm{sHA}$ in the tumour context. Fragmented HA has been found in prostate and mammary tumour extracts, in urine from bladder cancer patients, in serum from renal cancer patients and in saliva from head and neck cancer patients (Kumar et al, 1989; Lokeshwar et al, 1997, 2001; Franzmann et al, 2003; Koyama et al, 2007). These studies were limited to individual tumour samples, and the size and concentration of sHA was not addressed. Thus, a functional role for sHA in the tumour context in vivo remains largely speculative.

A robust method for isolating TIF has recently been established (Wiig et al, 2003). By extending this method, we describe here an assay for the differential quantification of total HA and sHA in TIF. While all TIF samples contained HA, only a proportion of TIFs contained detectable levels of sHA. These concentrations reached up to $6 \mu \mathrm{g} \mathrm{ml}^{-1}$, at which biological effects are exerted (West et al, 1985; Koyama et al, 2007; Matou-Nasri et al, 2009). Prospective studies with human colorectal tumours showed that high sHA concentrations in TIF correlate with lymphatic vessel invasion and lymph node metastasis. Together, these results suggest that sHA can accumulate in TIF and foster tumour progression.

\section{MATERIALS AND METHODS}

Preparation of hyaluronan oligosaccharides. Ultrapure HMWHA (Healon 5) from Amo (Ettlingen, Germany) was dissolved at

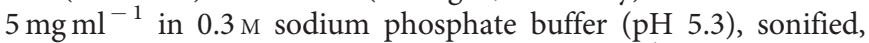
then enzymatically digested with $200 \mathrm{Uml}^{-1}$ bovine testis hyaluronidase (Sigma-Aldrich, Taufkirchen, Germany) for $6 \mathrm{~h}$ at $37^{\circ} \mathrm{C}$. The resulting fragments were separated on a Bio Gel P10 column $(3.5 \times 115 \mathrm{~cm})$ (BioRad, München, Germany) and $3 \mathrm{ml}$ fractions were collected. Hyaluronic acid concentrations in the fractions were determined by measuring the absorbance at $210 \mathrm{~nm}$ with reference to standards, or using an HA ELISA-like assay (Echelon, Salt Lake City, UT, USA). Fluorophore-assisted carbohydrate electrophoresis (FACE) analysis of ANDS-labelled fragments was performed as described (Termeer et al, 2000). Hyaluronic acid fragments of defined size (Oligo $\mathrm{HA}_{6}$, SigmaAldrich) served as reference standards. Hyaluronic acid with a molecular weight of $25-75 \mathrm{kDa}$ (intermediate-sized HA) and Streptomyces hyaluronidase were from Sigma.

Cell lines and tumour cell injection. The cultivation, derivation and tumour morphology of the rat tumour cell lines used in this study (BSp73-1AS, BSp73-10AS and BSp73-ASML (pancreatic carcinomas), NM-081, MT-450 and MTLy (mammary adenocarcinomas), AT3.1 and MatLu (prostate adenocarcinomas), NID2 (neu-transformed Schwannoma), and BDX2 (fibrosarcoma)) has been described previously (Nestl et al, 2001; Krishnan et al, 2003). BSp73-1AS, NM-081 and BDX2 are non-metastatic, whereas the other cell lines are metastatic to varying degrees in syngeneic animals (Nestl et al, 2001; Krishnan et al, 2003). Tumour cells $\left(1 \times 10^{6}\right.$ in PBS $)$ were injected subcutaneously into the appropriate syngeneic rat strain. Animals were killed when tumours reached the legal size limit of $5 \mathrm{~cm}$ in one direction or when the animals became moribund. Excised tumours were immediately processed on ice or snap-frozen. All animal experiments were approved by the local regulatory authorities.

Production of TIF and tumour lysates. Interstitial fluid was prepared as described (Wiig et al, 2003). Briefly, tissue samples were cut into small pieces, placed in a $40-\mu \mathrm{M}$ nylon cell strainer (BD biosciences, Heidelberg, Germany) on top of a $50-\mathrm{ml}$ centrifuge tube (Greiner Bio-One, Frickenhausen, Germany), and centrifuged at $400 \mathrm{~g}$ for 1 hour at $4{ }^{\circ} \mathrm{C}$. To separate sHA from HMW-HA, interstitial fluid was centrifuged through Amicon Ultracentrifugal Filters with a molecular weight cutoff (MWCO) of $10 \mathrm{kDa}$ (Millipore, Billerica, MA, USA). For tumour lysate preparation, tumour samples were homogenised in lysis buffer (30 mm Tris ( $\mathrm{pH} 7.5$ ), $150 \mathrm{~mm} \mathrm{NaCl}, 5 \mathrm{~mm}$ EDTA, 0.5\% NP40 and protease inhibitors) and clarified by centrifugation.

Patient tumour samples. Primary tumours excised from cancer patients being treated at Universitätsmedizin Mannheim or St Vincentiuskliniken Karlsruhe were used in this study. The procedures were approved by the ethics committee at the Universitätsmedizin Mannheim (2013-840R-MA) and carried out in accordance with the Helsinki Declaration. Samples were either snap-frozen or processed immediately for TIF isolation. The presence of lymph node metastases and invasion into lymphatic vessels was documented by a qualified pathologist $(A D)$ as part of routine pathological analysis.

\section{RESULTS}

A method for the detection and quantification of sHA in TIF. To determine the pathophysiological concentration of sHA in tumours, we established a method for the quantification of sHA in TIF (Figure 1). In this method, TIF is collected using established methods (Wiig et al, 2003), then the sHA in the TIF is separated from higher-molecular-weight HA using ultracentrifugal filters. Subsequently, the concentration of sHA in the flow-through is analysed by an ELISA-like assay that employs a HA-binding protein.

First, we determined the appropriate ultracentrifugal filter to use. We calculated that ultracentrifugal filters with a MWCO of $10 \mathrm{kDa}$ should allow sHA of $25 \mathrm{HA}$ disaccharides or smaller to pass through. To verify this, we partially digested HMW-HA with 
hyaluronidase to produce a mixture of HA oligosaccharides differing in length by one repeat disaccharide (Figure 2A, lane 1). Centrifugation of this $\mathrm{HA}$ digest through the ultracentrifugal filters and subsequent FACE analysis of the flow-through confirmed that the majority of HA fragments present in the

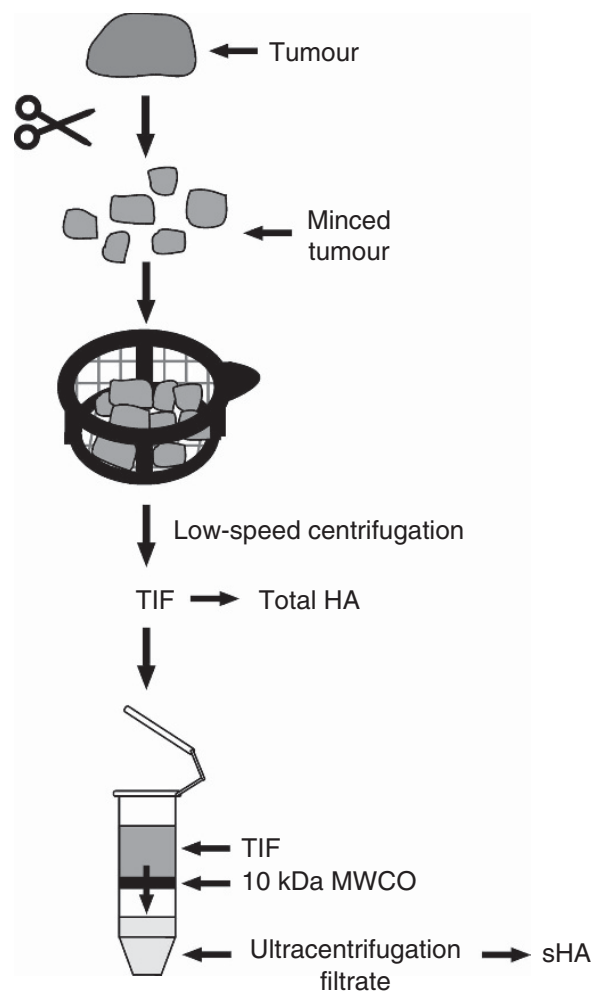

Figure 1. Schematic overview of the method for isolating and quantifying sHA from TIF. Small tumour pieces are placed in a $40-\mu \mathrm{M}$ nylon cell strainer and TIF is collected by low-speed centrifugation. Ultracentrifugal filters are used to separate sHA smaller than $10 \mathrm{kDa}$ in the TIF from larger HA molecules. The total HA concentration in TIF or the sHA concentration in the ultracentrifugation filtrate is determined by the HA ELISA-like assay. filtrate are 25 disaccharides in length or less, with only very low quantities of fragments larger than 25 disaccharides passing through the filter (Figure 2A).

The ELISA-like assay detects both HMW-HA and a mixture of sHA fragments (Figure 2B). To determine whether sHA size affects the linearity of the assay, we collected sHA fragments of specific chain length by fractionating a partial hyaluronidase HA digest using size-exclusion chromatography. Equimolar concentrations of HA fragments of defined size were then analysed using the ELISAlike assay. As shown in Figure 2C, the ELISA-like assay can detect HA fragments of six or more disaccharides in a linear manner.

In further control experiments with ultracentrifugal filtration of intermediate-sized HA $(25-75 \mathrm{kDa})$, no HA was found in the filtrate but was fully recovered from the retentate (Supplementary Figure 1). These data confirm the size selectivity of the ultracentrifugal filters, demonstrate that the centrifugation process does not degrade HA into oligosaccharides, and show that the filter does not retain HA. Thus, the amount of HA in both the filtrate and the retentate can be accurately quantified. The method depicted in Figure 1 therefore allows quantification of sHA fragments of 6-25 disaccharides.

TIF from experimental tumours contains HMW-HA and variable amounts of sHA. To determine the amount of HA in TIF, we first analysed TIF isolated from freshly excised rat tumours for total $\mathrm{HA}$ as well as sHA content (Figure 3A). Total HA concentrations were highly variable and ranged from 40 to $800 \mu \mathrm{g} \mathrm{ml}^{-1}$. In some TIF samples we could also detect sHA, although at much lower concentrations than total HA levels (Figure 3A). This suggests that most of the HA in TIF is above 25 disaccharides in length, but that accumulation of sHA can occur in some tumours. To assess variability in the presence and concentration of sHA, we examined 10 different syngeneic rat tumour models. In each case, TIF taken from multiple tumourbearing animals was analysed (Figure 3B). In each tumour model in which sHA could be detected, sHA was consistently present in TIF from all animals. This suggests that sHA levels in TIF are determined by the specific properties of the tumour.

As HA is continuously produced and degraded physiologically (Laurent and Fraser, 1992), we investigated whether the accumulation of sHA is specific for interstitial fluid from tumours, or is also
A

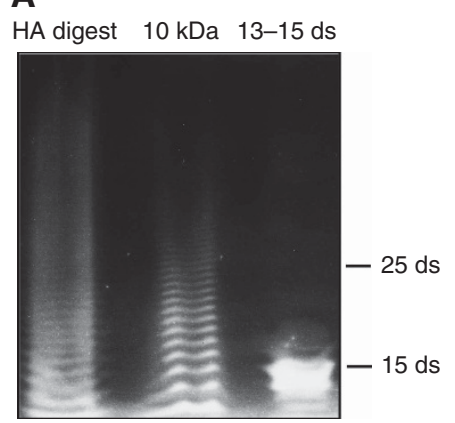

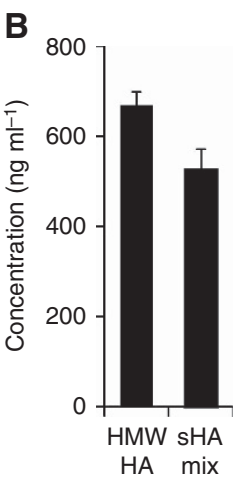

Figure 2. Characterisation of the ultracentrifugal filters and the HA ELISA-like assay used for determining sHA concentrations in TIF. (A) A digest of HMW-HA containing a mixture of HA fragments was prepared (HA digest) and centrifuged through a $10 \mathrm{kDa}$ MWCO ultracentrifugal filter $(10 \mathrm{kDa})$. Fluorophore-assisted Carbohydrate electrophoresis analysis was used to detect the HA fragments before and after centrifugation. Purified HA fragments of defined size (13-15 disaccharides) were used as reference standards. The numbers on the right of the figure indicate the length of the indicated HA oligosaccharides. Ds: disaccharides. (B) A digest of HMW-HA containing a mixture of HA fragments was prepared and centrifuged through a 10-kDa MWCO ultracentrifugal filter (sHA mix). The HA ELISA-like assay was used to quantify the concentration of the sHA mix in comparison to an equivalent amount of undigested HMW-HA. (C) Hyaluronic acid fragments of defined size were purified from a HA digest using size-exclusion chromatography. Equivalent molarities $(250 \mathrm{~nm})$ of HA fragments of defined length were analysed using the HA ELISA-like assay. The apparent concentration of the samples as measured in the ELISA-like assay is presented. The size of the HA oligosaccharides measured is indicated in disaccharides. Buffer served as a control. 

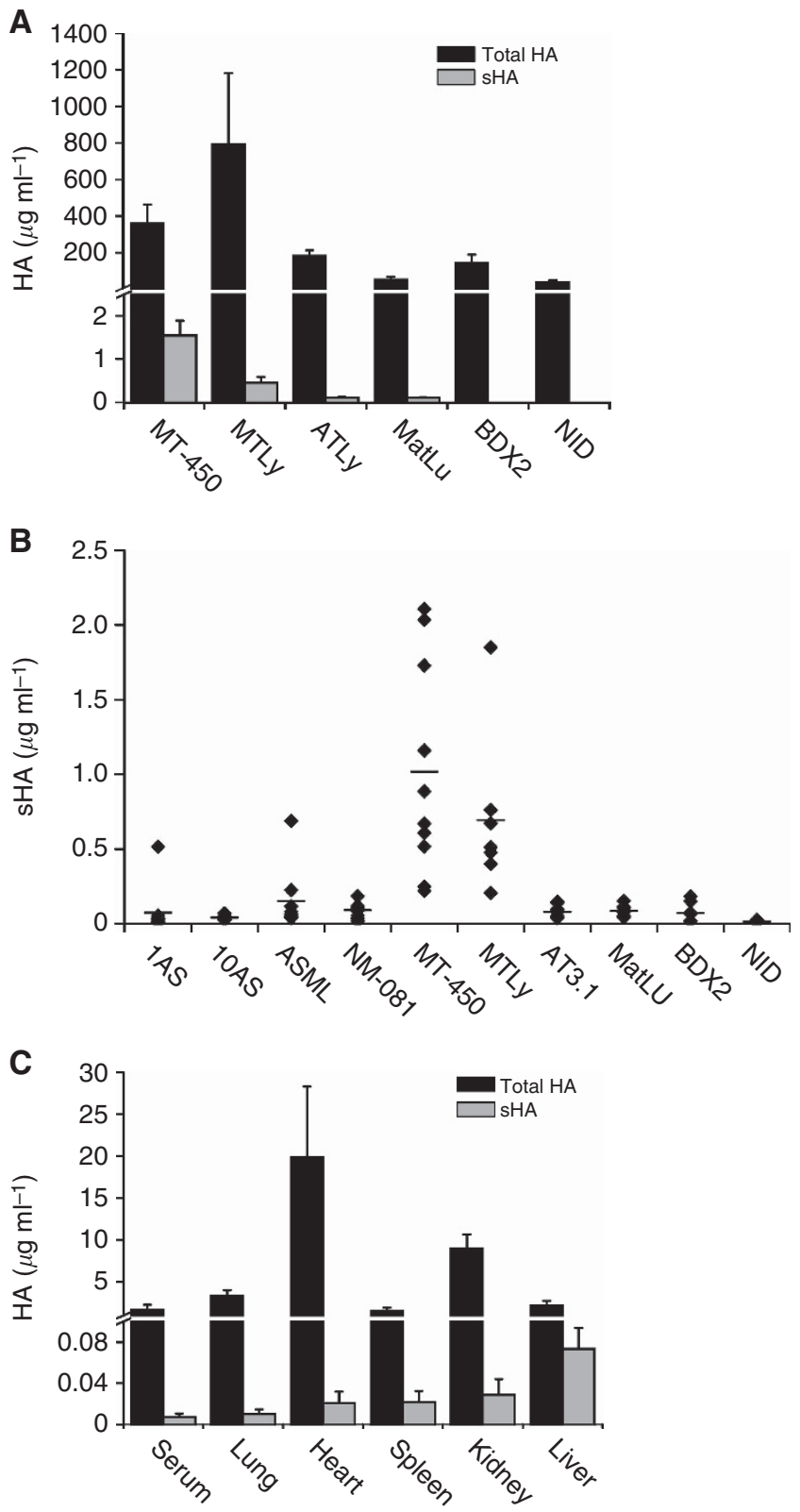

Figure 3. Assessment of total HA and sHA levels in TIF from syngeneic rat tumours and normal tissues. (A, B) TIF was isolated by low-speed centrifugation from the indicated freshly excised rat tumours. For separation of sHA from HMW-HA, TIF was centrifuged through 10-kDa MWCO ultracentrifugal filters. Using the HA ELISA-like assay the concentration of total HA was measured in the TIFs, and the concentration of sHA was measured in the ultracentrifugation filtrates. (A) The mean of total HA and sHA concentrations for three independent tumours for each of the indicated tumour models is shown together with the SE. (B) sHA concentrations in a panel of rat tumour models. Between 7 and 10 independent tumour-bearing animals were analysed for each tumour model. Each data point represents the sHA concentration in the TIF from one tumour. Bars represent the mean of all data from one group. (C) Assessment of physiological total $\mathrm{HA}$ and sHA levels in interstitial fluid of rat organs and in rat sera. Interstitial fluid was isolated from the indicated freshly excised rat organs. To separate sHA from HMW-HA, the interstitial fluid or sera were centrifuged through $10-\mathrm{kDa}$ MWCO ultracentrifugal filters. The concentration of total HA in the interstitial fluid or sera, as well as the concentration of sHA in the ultracentrifugation filtrates was measured using the HA ELISA-like assay. The mean of total $\mathrm{HA}$ and sHA concentrations for six independent samples for each organ or sera is shown together with the SE. found in interstitial fluid from organs or sera from healthy rats (Figure 3C). In all samples analysed, sHA levels were very low. Substantial total HA levels were observed, albeit at lower concentrations compared with those in TIF.

To confirm the specificity of the ELISA-like assay, we incubated MT-450 and MTLy TIF with Streptomyces hyaluronidase. Subsequently sHA levels measured by the ELISA-like assay were dramatically reduced in the TIF samples (Supplementary Figure 2A). To investigate whether components in TIF interfere with sHA detection in the ELISA-like assay, a defined amount of purified sHA was added to MatLu TIF that contains low endogenous sHA levels. Equivalent sHA concentrations in the TIF containing exogenously added sHA compared with the purified input sHA control were observed (Supplementary Figure 2B), demonstrating that sHA levels can be measured reliably in TIF.

To rule out the possibility that sHA is produced artificially during TIF workup in vitro, we added HMW-HA exogenously to 1AS, 10AS and ASML tumours, then prepared TIF and determined sHA levels (Supplementary Figure 2C). Compared with the signal obtained with the input HMW-HA, the sHA levels in the TIF from the HMW-HA-supplemented tumours were much lower, and similar to the endogenous levels observed in the TIF from these tumours (Figure $3 \mathrm{~B}$ ), indicating the sHA is not produced due to degradation of HMW-HA during TIF production.

Lysis of tumour samples released intracellular sHA (Supplementary Figure 2D), raising the possibility that sHA in TIF may be derived from intracellular sources due to limited cell lysis. However, this is unlikely for a number of reasons. Tracer experiments indicate that the method we employed produces TIF that contains fluids derived exclusively from the extracellular space and not from intracellular sources (Wiig et al, 2003). Furthermore, we found that TIF from many tumour samples did not contain sHA (Figure 3B), suggesting that cell lysis and release of sHA is not an integral feature of the TIF preparation method, underscoring the notion that cell lysis contributes little if any to the sHA found in TIF.

Next, we characterised the molecular size distribution of HA degradation products in TIF. As FACE analysis was not possible due to impurities in TIF, we performed size-exclusion chromatography of TIF from MTLy and MT-450 rat tumours with subsequent analysis of HA size and concentration in the fractions. TIF from both tumour types contained sHA oligosaccharides of different sizes (Figure 4). In MTLy TIF, most sHA was larger than 13 disaccharides, whereas most of the sHA in MT-450 TIF was present in four peaks representing sHA fragments of six to nine disaccharides. FACE analysis of selected chromatography fractions confirmed the size of HA oligosaccharides in specific fractions (Supplementary Figure 3).

Levels of sHA in TIF from human colorectal tumours correlate with lymphatic vessel invasion and lymph node metastasis. To investigate whether human tumours also contain sHA, we isolated TIF from fresh primary tumour samples obtained from patients suffering from a variety of cancer types. Similar to the case with the rat tumours, we found HA in all TIFs analysed. A subset of TIFs also contained sHA, albeit at much lower concentrations than the total $\mathrm{HA}$, with sHA concentrations up to $6 \mu \mathrm{g} \mathrm{ml}^{-1}$ being detected (Figure $5 \mathrm{~A}$ and $\mathrm{B}$ ).

Retrospective studies would be facilitated if frozen archived human tumour material could be used reliably for the isolation of TIF and the sHA therein. First, we compared the sHA content of TIF prepared from fresh and snap-frozen samples taken from the identical experimental tumour. For MT-450 and MTLy tumours it was equivalent (Figure 5C). TIF from BDX2 and NM-081 tumours prepared by both methods contained very low sHA concentrations. We then analysed frozen human tumours. While HA was present 

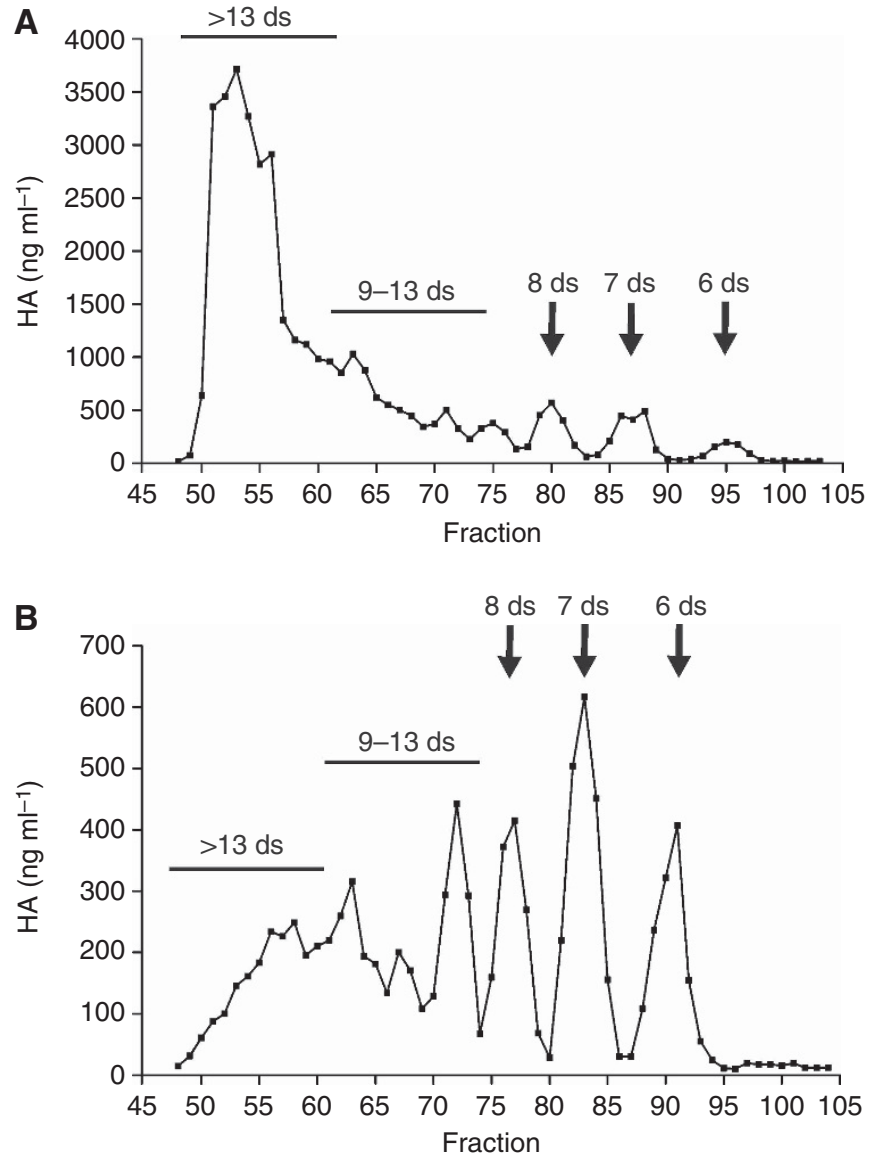

Figure 4. The profile of sHA fragments contained in TIF from MTLy (A) and MT-450 (B) tumours. Tumour interstitial fluid from multiple MT-450 or MTLy tumours was pooled and subjected to ultracentrifugal filtration through 10-kDa Amicon filters. The filtrates were concentrated in a Speed Vac, then applied to a Bio Gel P10 size-exclusion

chromatography column $(3.5 \times 115 \mathrm{~cm})$ and eluted with $0.3 \mathrm{M}$ sodium phosphate buffer ( $\mathrm{pH}$ 5.3). The HA concentrations in the eluted $3 \mathrm{ml}$ fractions were determined by the HA ELISA-like assay. The column was calibrated by fractionating a partial HA digest under identical conditions, then determining the size of the HA oligosaccharides in the fractions using FACE analysis. The region in which HA fragments of a specific length were eluted is indicated in the diagram.

in the TIF from all tumours, a subset of samples contained sHA concentrations up to $4.5 \mu \mathrm{g} \mathrm{ml}^{-1}$ (Figure 5D), comparable to those obtained with fresh material. These data suggest that sHA can be reliably measured in TIF from frozen tumour samples.

Concentrations of sHA above $500 \mathrm{ng} \mathrm{ml}^{-1}$ have been implicated in a number of processes that contribute to tumour growth and dissemination. In prospective studies, we therefore compared sHA concentrations in TIF from 72 colorectal cancer patients with clinicopathological factors. The characteristics of the cohort used are detailed in Supplementary Table 1. Concentrations of sHA of $>500 \mathrm{ng} \mathrm{ml}^{-1}$ were found in $\sim 20 \%$ of tumours, but did not correlate with tumour size, histological grade, or blood vessel invasion (data not shown). However, patients whose TIF contained $>500 \mathrm{ng} \mathrm{ml}^{-1} \mathrm{sHA}$ had a statistically significant higher incidence of lymph node metastasis (Figure 6A and C). Consistently, invasion of tumour cells into the lymphatic vessels (Figure 6B) was observed more frequently in a statistically significant manner in tumours from patients whose TIF contained sHA concentrations above $500 \mathrm{ng} \mathrm{ml}^{-1}$ (Figure 6D).
DISCUSSION

Although sHA has the potential to influence tumour growth and progression, and HA degradation products have been detected in individual tumours (Kumar et al, 1989; Lokeshwar et al, 2001; Franzmann et al, 2003; Koyama et al, 2007), the pathophysiological concentration of sHA in the tumour microenvironment has not been determined. Here we describe a novel method for determining the concentration of HA fragments ranging from 6 to 25 disaccharides in TIF. While most HA present in TIF was above 25 disaccharides in size, concentrations of sHA up to $6 \mu \mathrm{g} \mathrm{ml}^{-1}$ were found in a subset of tumours. In prospective studies we found that colorectal cancer patients with sHA levels above $500 \mathrm{ng} \mathrm{ml}^{-1}$ in their TIF are more likely to have lymph node metastases and lymphatic vessel invasion, suggesting that pathophysiological concentrations of sHA might contribute to tumour progression.

The association between enhanced sHA levels in TIF, tumour cell invasion into the lymphatic vessels and lymph node metastasis formation may speak for a functional role for sHA in tumour progression. The concentrations of sHA we found in some TIFs exert a number of relevant biological effects. For example, sHA can increase the expression or activity of certain matrix metalloproteases, and in some studies this effect was observed at the sHA concentrations found in TIF (David-Raoudi et al, 2008; MatouNasri et al, 2009). It has also been suggested to be an autocrine motility factor (Saito et al, 2011). Furthermore, sHA can influence angiogenesis (Simpson and Lokeshwar, 2008). The angiogenic effects of sHA are limited to oligosaccharides of 4-25 disaccharides in length (West et al, 1985), with sHA concentrations of $0.5 \mu \mathrm{g} \mathrm{ml}^{-1}$ promoting migration and tubule formation of endothelial cells in vitro (West and Kumar, 1989; Lokeshwar and Selzer, 2000; Matou-Nasri et al, 2009). In vivo, sHA can stimulate neovascularisation in matrigel plug assays. The strongest effect was observed at $1 \mu \mathrm{g} \mathrm{ml}^{-1}$, while concentrations of $\geqslant 50 \mu \mathrm{g} \mathrm{ml}^{-1}$ inhibited angiogenesis (Koyama et al, 2007). Pro-tumourigenic effects of HA oligosaccharides in the sHA range have also been reported. For example, a 12-disaccharide HA fragment injected intratumourally promoted growth of experimental tumours (Dang et al, 2013). Hyaluronan of 18 disaccharides in length promoted cleavage of CD44 and migration of cancer cells, while intermediate-sized HA fragments of $>36 \mathrm{kDa}$ (90 disaccharides in length) did not (Sugahara et al, 2003). Thus, the size and concentration of sHA we found in some TIF samples are clearly in the pro-angiogenic and pro-tumourigenic range. Given the association between sHA levels, lymphatic invasion and lymph node metastasis we report here, future work will explore the effects of sHA on lymphangiogenesis. As tumour-induced lymphangiogenesis can promote metastasis to lymph nodes (Sleeman et al, 2009), such a mechanism may also help to explain the association we report here between sHA levels and the incidence of lymph node metastases in human colorectal cancer patients.

Molecularly, several cell surface receptors have been implicated in mediating the biological activities of sHA, all of which bind to both sHA and HA fragments larger than 25 disaccharides in length. CD44, the main cell surface HA receptor, binds to HA of six disaccharides and longer (Lesley et al, 2000), and mediates sHA-induced activation of signalling pathways in endothelial cells (Slevin et al, 2007; Matou-Nasri et al, 2009). Another HA receptor, RHAMM, has a similar role in sHA-induced signal transduction in endothelial cells (Lokeshwar and Selzer, 2000) and angiogenesis (Gao et al, 2008). Toll-like receptors such as TLR-4 have also been shown to transduce sHA signalling, for example, in dendritic cells (Termeer et al, 2002) and endothelial cells (Taylor et al, 2004). Crosstalk between these sHA receptors has also been reported (Bourguignon et al, 2011). 

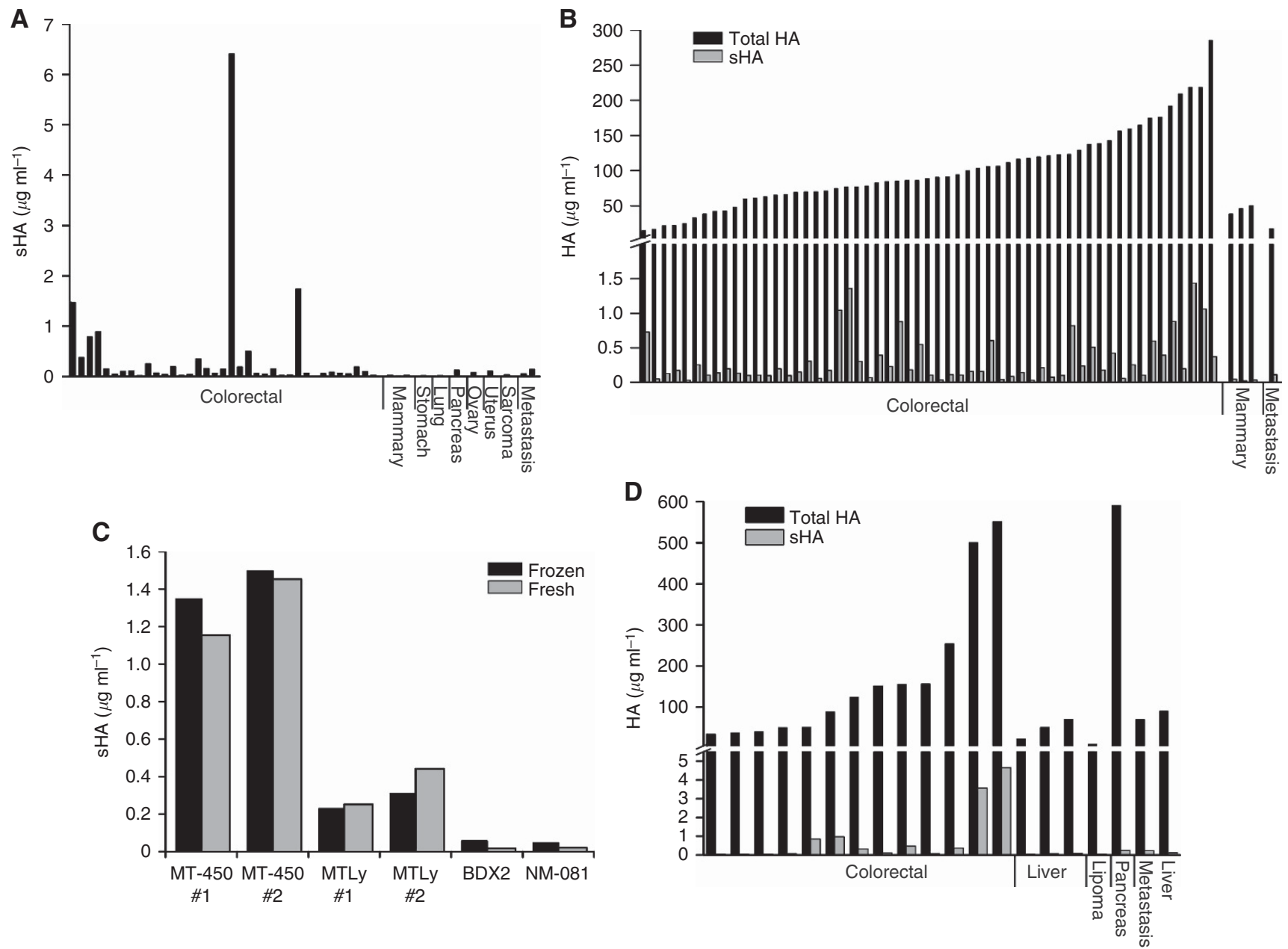

Figure 5. Assessment of total HA and sHA levels in TIF from human tumours. (A, B) TIF was isolated from the indicated human tumours immediately following surgical removal, and ultracentrifugation filtrates were prepared. The concentration of total HA in the TIFs and the sHA concentration in the ultracentrifugation filtrates was determined using the HA ELISA-like assay. Each bar represents the total HA or sHA concentration in the TIF from one individual tumour as indicated. (C) The indicated independent rat tumours were divided in half. One half was snap-frozen, then thawed, and the other half was used as a fresh tumour tissue. TIF and ultracentrifugation filtrates were prepared and the sHA concentration in both preparations of TIF was determined using the HA ELISA-like assay. (D) Snap-frozen tumour samples from human patients were thawed and TIF prepared. Total HA concentrations and sHA concentrations were analysed in TIF or in ultracentrifugation filtrates, respectively. Each bar represents the total HA or sHA concentration from one individual tumour as indicated.

The molecular basis for the size-specific functional differences between sHA and larger HA molecules remains to be elucidated. The definition of the critical functional size of HA oligosaccharides (sHA), and low-, intermediate- and high-molecular-weight HA in the literature is arbitrary and poorly defined, but is nevertheless pivotal if size-specific functional differences between these differently sized HA molecules are to be understood. Here we have defined sHA as 6-25 disaccharides in length due to the parameters defined by our experimental system, which corresponds to the size range that promotes angiogenesis (West et al, 1985). Nevertheless, HA fragments larger than 25 disaccharides have also been reported to exert different biological effects compared with HMW-HA, for example, in promoting tumour cell motility (Jiang et al, 2011). Importantly, we note that monovalent binding of HA by CD44 is observed in the range of 6-18 disaccharides (Lesley et al, 2000), and this is likely to be decisive for sHA-specific functions. Thus size-specific functional differences may be accounted for by the fact that HMW-HA induces CD44 clustering due to multivalent binding, which can be attenuated by sHA (Yang et al, 2012). Accordingly, sHA can interfere with the HMW-HA-dependent co-receptor function of CD44 in the context of angiogenesis (Fuchs et al, 2013). On the other hand, sialylation of CD44 inhibits its ability to bind to HA through a mechanism thought to involve steric hinderance due to the negative charge on HA molecules (Jackson, 2009). Conceivably, sHA may be able to circumvent such steric hinderance due to its small size, which could potentially provide an alternative explanation for the size-dependent effects of HA.

The process that leads to the generation and accumulation of sHA remains uncertain. Currently, we cannot distinguish whether the sHA that accumulates in TIF results from degradation of HMW-HA, or from synthesis of low-molecular-weight HA. However, the properties of the HA synthases (Itano et al, 1999a) suggest that sHA likely accumulates due to degradation of HMWHA. The small size of sHA means it will be rapidly cleared, in turn requiring relatively large amounts of HMW-HA as a prerequisite for the accumulation of sHA in tumours. Accordingly, enhanced concomitant synthesis and degradation of HA in tumours has the most pronounced effect on tumour growth and metastasis (Enegd et al, 2002; Simpson, 2006; Bharadwaj et al, 2009). Consistently, we 
A
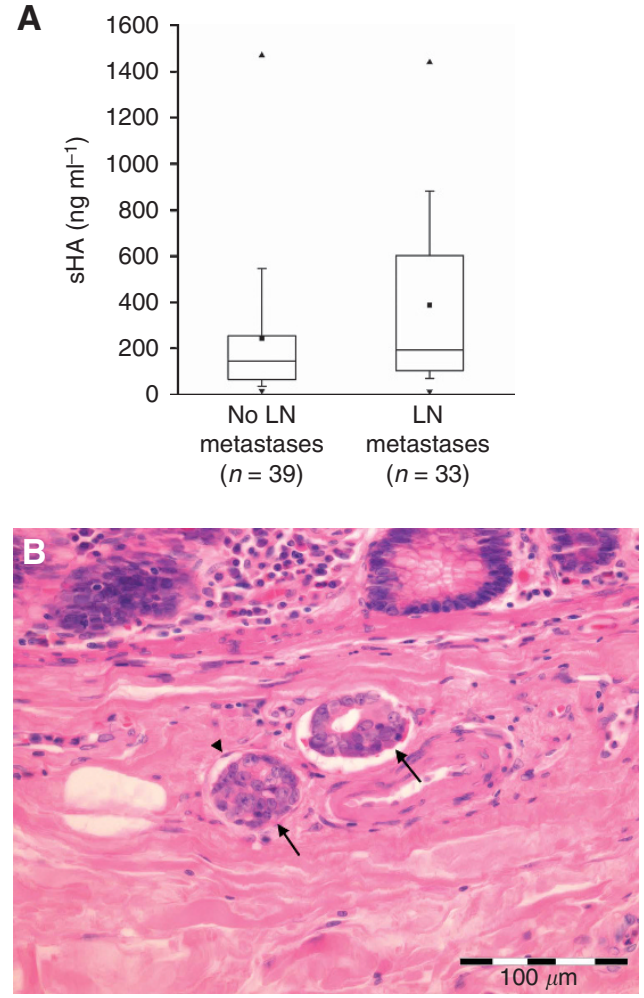

c

\begin{tabular}{|c|c|c|}
\hline & $>500 \mathrm{ng} \mathrm{ml}^{-1} \mathrm{sHA}$ & $<500 \mathrm{ng} \mathrm{m}^{-1} \mathrm{sHA}$ \\
\hline No LN metastases & $4 / 39(10 \%)$ & $35 / 39(90 \%)$ \\
\hline LN metastases & $11 / 33(33 \%)$ & $22 / 33(67 \%)$ \\
\hline \multicolumn{2}{|c|}{$P=0.0211$}
\end{tabular}

D

\begin{tabular}{|c|c|c|}
\hline & $>500 \mathrm{ng} \mathrm{ml}^{-1} \mathrm{sHA}$ & $<500 \mathrm{ng} \mathrm{ml}^{-1} \mathrm{sHA}$ \\
\hline No LN invasion & $5 / 42(12 \%)$ & $37 / 42(88 \%)$ \\
\hline LN invasion & $9 / 21(43 \%)$ & $12 / 21(57 \%)$ \\
\hline \multicolumn{2}{|c|}{$P=0.0093$}
\end{tabular}

Figure 6. Correlation between sHA concentrations in TIF from human cancer patients, and the presence of regional lymph node metastases and lymphatic vessel invasion. Tumour interstitial fluid (TIF) was isolated from 72 human colorectal tumours immediately following surgical removal. Ultracentrifugation filtrates were prepared and the sHA concentration was determined using the HA ELISA-like assay. The presence of lymph node metastases was evaluated using the TNM staging system, where the staging $\mathrm{NO}$ indicated no presence of metastases in the regional lymph nodes, and patients with N1a, N1b, N2a and N2b had lymph node metastases. (A) Box-plot comparing the distribution of sHA concentrations in TIF from patients with and without lymph node metastases. The lower boundary of the box indicates the 25th percentile, the upper boundary of the box indicates the 75th percentile. The line within the box represents the median and the dot within the box indicates the mean. Whiskers above and below the boxes indicate the 90th and 10th percentile. Dots lying outside the whiskers represent the maximum and minimum of sHA levels detected. (B) Representative haematoxylin and eosin-stained tumour section showing the invasion of tumour cells into lymphatic vessels. Tumour cell clusters (arrows) are present inside lymphatic vascular spaces lined by endothelial cells (arrowhead). (C, D) Patients were classified as having either high and low sHA concentrations in their primary tumours (cutoff $500 \mathrm{ng} \mathrm{ml}^{-1} \mathrm{sHA}$ ), and then grouped according to whether or not lymph node metastasis (C) or lymphatic vessel invasion (D) was present. Statistical analysis using Fisher's test shows that both lymph node metastasis and lymphatic vessel invasion correlates significantly with the presence of high sHA concentrations $(P=0.0211$ (C) and $P=0.0093$ (D)). found that HMW-HA was found in all TIF samples analysed, while sHA-when detectable-was present at much lower concentrations. Furthermore, we observed lower total HA levels in interstitial fluid from normal tissues, and no significant levels of sHA.

The catabolism of HA is poorly understood (Stern, 2008). Free radicals have the potential to depolymerise HA (Soltés et al, 2006; Stern et al, 2007), but may act indirectly in vivo (Monzon et al, 2010), and the degree to which they might contribute to the production of sHA in tumours is uncertain. Several hyaluronidases can be expressed in tumours (Lokeshwar et al, 2001; Posey et al, 2003; Ekici et al, 2004; Paiva et al, 2005; Udabage et al, 2005; Tan et al, 2011). Of these, the extracellular location and enzymatic properties of Hyal-1 (Frost et al, 1997; Harada and Takahashi, 2007) would be consistent with a role in the production of sHA. Interestingly, Hyal-1 is expressed in prostate tumours that contain HA oligosaccharides (Lokeshwar et al, 2001), and its expression has been associated with poor prognosis for a number of types of cancer (Ekici et al, 2004; Poola et al, 2008; Gomez et al, 2009; Kramer et al, 2010). Furthermore, ectopic expression of Hyal-1 promoted lymph node metastasis (Patel et al, 2002; Kovar et al, 2006; Bharadwaj et al, 2009), while inhibition of Hyal-1 activity in a prostate cancer model reduced tumour growth and angiogenesis (Benitez et al, 2011). Nevertheless, direct evidence for the involvement of Hyal-1 in the local degradation of HA and accumulation of sHA is lacking.

In addition to the tumour context, sHA can accumulate in other pathological conditions. Accumulation of HA has been observed in fluids collected from injured tissues, such as bleomycin-injured lungs and experimental wounds (Savani et al, 2000; Taylor et al, 2004), and breakdown of HA has been reported after tissue injury (Chajara et al, 2000; Al'Qteishat et al, 2006) and during inflammation (Campo et al, 2012). An inflammatory microenvironment is often an important component of tumours (Grivennikov et al, 2010), and may therefore contribute to HA degradation and accumulation of sHA in the context of tumours. Furthermore, as sHA has pro-inflammatory and immunostimulatory properties (Termeer et al, 2000, 2002; Taylor et al, 2004; David-Raoudi et al, 2008), sHA may influence wound healing and inflammatory processes, in addition to contributing to the inflammatory status of tumours. In future work we plan to use the method described here to study the significance and function of sHA in the context of tissue damage and inflammation.

Determination of sHA levels in TIF may have prognostic value. Notably, high sHA concentrations in TIF from colorectal cancer patients correlated with the presence of lymph node metastases and lymphatic vessel invasion, two important prognostic factors for colorectal cancer (Minsky et al, 1989; Leong et al, 2007; Lin et al, 2010). In further work we will follow up these patients to determine whether sHA levels predict survival. The analysis of sHA levels in archived frozen tumour material will also allow sHA levels to be correlated with patient survival in retrospective studies. For prostate and bladder cancer, we note that the measurement of total HA levels and hyaluronidase activity in urine is reported to be a sensitive and accurate marker for diagnosis and prognosis (Lokeshwar et al, 1996; Pham et al, 1997; Ekici et al, 2004; Simpson and Lokeshwar, 2008). Measuring sHA levels in TIF has advantages over these parameters and may thus improve sensitivity and accuracy, because the effector molecules are measured directly in the context in which they can exert biological activity.

In summary, the novel assay for measuring sHA levels in interstitial fluid we describe here has allowed us for the first time to determine pathophysiologically relevant sHA concentrations in the tumour context. These levels correlate with lymphatic vessel invasion and lymph node metastasis, providing a rationale for further studies to investigate whether sHA has a functional role in tumour progression, and whether the evaluation of sHA in TIF might have prognostic value. 


\section{ACKNOWLEDGEMENTS}

This work was supported by a grant to JPS (Deutsche Forschungsgemeinschaft Schwerpunkt Program SPP1190 (Tumour-vessel interface)) and by contract research Glykobiologie/Glykomik of the Baden-Württemberg Stiftung. We are grateful to Annette Gruber, Diana Plaumann-Ziegler, Gitta Flaig, and the staff of the rodent facility at KIT Karlsruhe for their excellent technical support.

CONFLICT OF INTEREST

The authors declare no conflict of interest.

\section{REFERENCES}

Al'Qteishat A, Gaffney J, Krupinski J, Rubio F, West D, Kumar S, Kumar P, Mitsios N, Slevin M (2006) Changes in hyaluronan production and metabolism following ischaemic stroke in man. Brain J Neurol 129: 2158-2176.

Anttila MA, Tammi RH, Tammi MI, Syrjänen KJ, Saarikoski SV, Kosma VM (2000) High levels of stromal hyaluronan predict poor disease outcome in epithelial ovarian cancer. Cancer Res 60: 150-155.

Auvinen P, Tammi R, Parkkinen J, Tammi M, Agren U, Johansson R, Hirvikoski P, Eskelinen M, Kosma VM (2000) Hyaluronan in peritumoral stroma and malignant cells associates with breast cancer spreading and predicts survival. Am J Pathol 156: 529-536.

Benitez A, Yates TJ, Lopez LE, Cerwinka WH, Bakkar A, Lokeshwar VB (2011) Targeting hyaluronidase for cancer therapy: antitumor activity of sulfated hyaluronic acid in prostate cancer cells. Cancer Res 71: 4085-4095.

Bharadwaj AG, Kovar JL, Loughman E, Elowsky C, Oakley GG, Simpson MA (2009) Spontaneous metastasis of prostate cancer is promoted by excess hyaluronan synthesis and processing. Am J Pathol 174: 1027-1036.

Bourguignon LYW, Wong G, Earle CA, Xia W (2011) Interaction of low molecular weight hyaluronan with $\mathrm{CD} 44$ and toll-like receptors promotes the actin filament-associated protein 110-actin binding and MyD88-NFkB signaling leading to proinflammatory cytokine/chemokine production and breast tumor invasion. Cytoskeleton (Hoboken) 68: 671-693.

Campo GM, Avenoso A, D'Ascola A, Scuruchi M, Prestipino V, Nastasi G, Calatroni A, Campo S (2012) The inhibition of hyaluronan degradation reduced pro-inflammatory cytokines in mouse synovial fibroblasts subjected to collagen-induced arthritis. J Cell Biochem 113: 1852-1867.

Chajara A, Raoudi M, Delpech B, Leroy M, Basuyau JP, Levesque H (2000) Increased hyaluronan and hyaluronidase production and hyaluronan degradation in injured aorta of insulin-resistant rats. Arterioscler Thromb Vasc Biol 20: 1480-1487.

Dang S, Peng Y, Ye L, Wang Y, Qian Z, Chen Y, Wang X, Lin Y, Zhang X, Sun X, Wu Q, Cheng Y, Nie H, Jin M, Xu H (2013) Stimulation of TLR4 by LMW-HA induces metastasis in human papillary thyroid carcinoma through CXCR7. Clin Dev Immunol 2013: 712561.

David-Raoudi M, Tranchepain F, Deschrevel B, Vincent J-C, Bogdanowicz P, Boumediene K, Pujol J-P (2008) Differential effects of hyaluronan and its fragments on fibroblasts: relation to wound healing. Wound Repair Regen Off Publ Wound Heal Soc Eur Tissue Repair Soc 16: 274-287.

Ekici S, Cerwinka WH, Duncan R, Gomez P, Civantos F, Soloway MS, Lokeshwar VB (2004) Comparison of the prognostic potential of hyaluronic acid, hyaluronidase (HYAL-1), CD44v6 and microvessel density for prostate cancer. Int J Cancer 112: 121-129.

Enegd B, King JAJ, Stylli S, Paradiso L, Kaye AH, Novak U (2002) Overexpression of hyaluronan synthase-2 reduces the tumorigenic potential of glioma cells lacking hyaluronidase activity. Neurosurgery 50: 1311-1318.

Fieber C, Baumann P, Vallon R, Termeer C, Simon JC, Hofmann M, Angel P, Herrlich P, Sleeman JP (2004) Hyaluronan-oligosaccharide-induced transcription of metalloproteases. J Cell Sci 117: 359-367.

Franzmann EJ, Schroeder GL, Goodwin WJ, Weed DT, Fisher P, Lokeshwar VB (2003) Expression of tumor markers hyaluronic acid and hyaluronidase (HYAL1) in head and neck tumors. Int J Cancer 106: 438-445.
Frost GI, Csóka AB, Wong T, Stern R, Csóka TB (1997) Purification, cloning, and expression of human plasma hyaluronidase. Biochem Biophys Res Commun 236: 10-15.

Fuchs K, Hippe A, Schmaus A, Homey B, Sleeman JP, Orian-Rousseau V (2013) Opposing effects of high- and low-molecular weight hyaluronan on CXCL12induced CXCR4 signaling depend on CD44. Cell Death Dis 4: e819.

Gao F, Liu Y, He Y, Yang C, Wang Y, Shi X, Wei G (2010) Hyaluronan oligosaccharides promote excisional wound healing through enhanced angiogenesis. Matrix Biol 29: 107-116.

Gao F, Yang CX, Mo W, Liu YW, He YQ (2008) Hyaluronan oligosaccharides are potential stimulators to angiogenesis via RHAMM mediated signal pathway in wound healing. Clin Investig Med 31: E106-E116.

Gomez CS, Gomez P, Knapp J, Jorda M, Soloway MS, Lokeshwar VB (2009) Hyaluronic acid and HYAL-1 in prostate biopsy specimens: predictors of biochemical recurrence. J Urol 182: 1350-1356.

Grivennikov SI, Greten FR, Karin M (2010) Immunity, inflammation, and cancer. Cell 140: 883-899.

Harada H, Takahashi M (2007) CD44-dependent intracellular and extracellular catabolism of hyaluronic acid by hyaluronidase- 1 and -2 . J Biol Chem 282: 5597-5607.

Itano N, Sawai T, Atsumi F, Miyaishi O, Taniguchi S, Kannagi R, Hamaguchi M, Kimata K (2004) Selective expression and functional characteristics of three mammalian hyaluronan synthases in oncogenic malignant transformation. J Biol Chem 279: 18679-18687.

Itano N, Sawai T, Miyaishi O, Kimata K (1999b) Relationship between hyaluronan production and metastatic potential of mouse mammary carcinoma cells. Cancer Res 59: 2499-2504.

Itano N, Sawai T, Yoshida M, Lenas P, Yamada Y, Imagawa M, Shinomura T, Hamaguchi M, Yoshida Y, Ohnuki Y, Miyauchi S, Spicer AP, McDonald JA, Kimata K (1999a) Three isoforms of mammalian hyaluronan synthases have distinct enzymatic properties. J Biol Chem 274: 25085-25092.

Jackson DG (2009) Immunological functions of hyaluronan and its receptors in the lymphatics. Immunol Rev 230: 216-231.

Jacobson A, Rahmanian M, Rubin K, Heldin P (2002) Expression of hyaluronan synthase 2 or hyaluronidase 1 differentially affect the growth rate of transplantable colon carcinoma cell tumors. Int J Cancer 102: 212-219.

Jiang D, Liang J, Noble PW (2011) Hyaluronan as an immune regulator in human diseases. Physiol Rev 91: 221-264.

Kosaki R, Watanabe K, Yamaguchi Y (1999) Overproduction of hyaluronan by expression of the hyaluronan synthase Has2 enhances anchorage-independent growth and tumorigenicity. Cancer Res 59: $1141-1145$.

Kovar JL, Johnson MA, Volcheck WM, Chen J, Simpson MA (2006) Hyaluronidase expression induces prostate tumor metastasis in an orthotopic mouse model. Am J Pathol 169: 1415-1426.

Koyama H, Hibi T, Isogai Z, Yoneda M, Fujimori M, Amano J, Kawakubo M, Kannagi R, Kimata K, Taniguchi S, Itano N (2007) Hyperproduction of hyaluronan in neu-induced mammary tumor accelerates angiogenesis through stromal cell recruitment: possible involvement of versican/PG-M. Am J Pathol 170: 1086-1099.

Koyama H, Kobayashi N, Harada M, Takeoka M, Kawai Y, Sano K, Fujimori M, Amano J, Ohhashi T, Kannagi R, Kimata K, Taniguchi S, Itano N (2008) Significance of tumor-associated stroma in promotion of intratumoral lymphangiogenesis: pivotal role of a hyaluronan-rich tumor microenvironment. Am J Pathol 172: 179-193.

Kramer MW, Golshani R, Merseburger AS, Knapp J, Garcia A, Hennenlotter J, Duncan RC, Soloway MS, Jorda M, Kuczyk MA, Stenzl A, Lokeshwar VB (2010) HYAL-1 hyaluronidase: a potential prognostic indicator for progression to muscle invasion and recurrence in bladder cancer. Eur Urol 57: 86-93.

Krishnan J, Kirkin V, Steffen A, Hegen M, Weih D, Tomarev S, Wilting J, Sleeman JP (2003) Differential in vivo and in vitro expression of vascular endothelial growth factor (VEGF)-C and VEGF-D in tumors and its relationship to lymphatic metastasis in immunocompetent rats. Cancer Res 63: 713-722.

Kumar S, West DC, Ponting JM, Gattamaneni HR (1989) Sera of children with renal tumours contain low-molecular-mass hyaluronic acid. Int J Cancer 44: 445-448.

Laurent TC, Fraser JR (1992) Hyaluronan. FASEB J Off Publ Fed Am Soc Exp Biol 6: 2397-2404.

Leong SPL, Cady B, Jablons DM, Garcia-Aguilar J, Reintgen D, Werner JA, Kitagawa Y (2007) Patterns of metastasis in human solid cancers. Cancer Treat Res 135: 209-221. 
Lesley J, Hascall VC, Tammi M, Hyman R (2000) Hyaluronan binding by cell surface CD44. J Biol Chem 275: 26967-26975.

Lin M, Ma S-P, Lin H-Z, Ji P, Xie D, Yu J-X (2010) Intratumoral as well as peritumoral lymphatic vessel invasion correlates with lymph node metastasis and unfavourable outcome in colorectal cancer. Clin Exp Metastasis 27: 123-132.

Lokeshwar VB, Lokeshwar BL, Pham HT, Block NL (1996) Association of elevated levels of hyaluronidase, a matrix-degrading enzyme, with prostate cancer progression. Cancer Res 56: 651-657.

Lokeshwar VB, Obek C, Soloway MS, Block NL (1997) Tumor-associated hyaluronic acid: a new sensitive and specific urine marker for bladder cancer. Cancer Res 57: 773-777.

Lokeshwar VB, Rubinowicz D, Schroeder GL, Forgacs E, Minna JD, Block NL, Nadji M, Lokeshwar BL (2001) Stromal and epithelial expression of tumor markers hyaluronic acid and HYAL1 hyaluronidase in prostate cancer. J Biol Chem 276: 11922-11932.

Lokeshwar VB, Selzer MG (2000) Differences in hyaluronic acid-mediated functions and signaling in arterial, microvessel, and vein-derived human endothelial cells. J Biol Chem 275: 27641-27649.

Matou-Nasri S, Gaffney J, Kumar S, Slevin M (2009) Oligosaccharides of hyaluronan induce angiogenesis through distinct CD44 and RHAMMmediated signalling pathways involving $\mathrm{Cdc} 2$ and gamma-adducin. Int J Oncol 35: 761-773.

Minsky BD, Mies C, Rich TA, Recht A (1989) Lymphatic vessel invasion is an independent prognostic factor for survival in colorectal cancer. Int J Radiat Oncol Biol Phys 17: 311-318.

Monzon ME, Fregien N, Schmid N, Falcon NS, Campos M, Casalino-Matsuda SM, Forteza RM (2010) Reactive oxygen species and hyaluronidase 2 regulate airway epithelial hyaluronan fragmentation. J Biol Chem 285: 26126-26134.

Nestl A, Von Stein OD, Zatloukal K, Thies WG, Herrlich P, Hofmann M, Sleeman JP (2001) Gene expression patterns associated with the metastatic phenotype in rodent and human tumors. Cancer Res 61: 1569-1577.

Paiva P, Van Damme M-P, Tellbach M, Jones RL, Jobling T, Salamonsen LA (2005) Expression patterns of hyaluronan, hyaluronan synthases and hyaluronidases indicate a role for hyaluronan in the progression of endometrial cancer. Gynecol Oncol 98: 193-202.

Patel S, Turner PR, Stubberfield C, Barry E, Rohlff CR, Stamps A, McKenzie E, Young K, Tyson K, Terrett J, Box G, Eccles S, Page MJ (2002) Hyaluronidase gene profiling and role of hyal-1 overexpression in an orthotopic model of prostate cancer. Int J Cancer 97: 416-424.

Pham HT, Block NL, Lokeshwar VB (1997) Tumor-derived hyaluronidase: a diagnostic urine marker for high-grade bladder cancer. Cancer Res 57: $778-783$.

Pirinen R, Tammi R, Tammi M, Hirvikoski P, Parkkinen JJ, Johansson R, Böhm J, Hollmén S, Kosma VM (2001) Prognostic value of hyaluronan expression in non-small-cell lung cancer: Increased stromal expression indicates unfavorable outcome in patients with adenocarcinoma. Int J Cancer 95: 12-17.

Poola I, Abraham J, Marshalleck JJ, Yue Q, Lokeshwar VB, Bonney G, Dewitty RL (2008) Molecular risk assessment for breast cancer development in patients with ductal hyperplasias. Clin Cancer Res 14: 1274-1280.

Posey JT, Soloway MS, Ekici S, Sofer M, Civantos F, Duncan RC, Lokeshwar VB (2003) Evaluation of the prognostic potential of hyaluronic acid and hyaluronidase (HYAL1) for prostate cancer. Cancer Res 63: 2638-2644.

Ropponen K, Tammi M, Parkkinen J, Eskelinen M, Tammi R, Lipponen P, Agren U, Alhava E, Kosma VM (1998) Tumor cell-associated hyaluronan as an unfavorable prognostic factor in colorectal cancer. Cancer Res 58: 342-347.

Saito T, Kawana H, Azuma K, Toyoda A, Fujita H, Kitagawa M, Harigaya K (2011) Fragmented hyaluronan is an autocrine chemokinetic motility factor supported by the HAS2-HYAL2/CD44 system on the plasma membrane. Int J Oncol 39: 1311-1320.

Savani RC, Hou G, Liu P, Wang C, Simons E, Grimm PC, Stern R, Greenberg AH, DeLisser HM, Khalil N (2000) A role for hyaluronan in macrophage accumulation and collagen deposition after bleomycininduced lung injury. Am J Respir Cell Mol Biol 23: 475-484.

Setälä LP, Tammi MI, Tammi RH, Eskelinen MJ, Lipponen PK, Agren UM, Parkkinen J, Alhava EM, Kosma VM (1999) Hyaluronan expression in gastric cancer cells is associated with local and nodal spread and reduced survival rate. Br J Cancer 79: 1133-1138.
Simpson MA (2006) Concurrent expression of hyaluronan biosynthetic and processing enzymes promotes growth and vascularization of prostate tumors in mice. Am J Pathol 169: 247-257.

Simpson MA, Lokeshwar VB (2008) Hyaluronan and hyaluronidase in genitourinary tumors. Front Biosci 13: 5664-5680.

Sironen RK, Tammi M, Tammi R, Auvinen PK, Anttila M, Kosma V-M (2011) Hyaluronan in human malignancies. Exp Cell Res 317: 383-391.

Sleeman J, Schmid A, Thiele W (2009) Tumor lymphatics. Semin Cancer Biol 19: 285-297.

Slevin M, Krupinski J, Gaffney J, Matou S, West D, Delisser H, Savani RC, Kumar S (2007) Hyaluronan-mediated angiogenesis in vascular disease: uncovering RHAMM and CD44 receptor signaling pathways. Matrix Biol 26: $58-68$.

Soltés L, Mendichi R, Kogan G, Schiller J, Stankovska M, Arnhold J (2006) Degradative action of reactive oxygen species on hyaluronan. Biomacromolecules 7: 659-668.

Stern R (2003) Devising a pathway for hyaluronan catabolism: are we there yet? Glycobiology 13: 105R-115R.

Stern R (2005) Hyaluronan metabolism: a major paradox in cancer biology. Pathol Biol (Paris) 53: 372-382.

Stern R (2008) Hyaluronidases in cancer biology. Semin Cancer Biol 18: 275-280.

Stern R, Asari AA, Sugahara KN (2006) Hyaluronan fragments: an information-rich system. Eur J Cell Biol 85: 699-715.

Stern R, Kogan G, Jedrzejas MJ, Soltés L (2007) The many ways to cleave hyaluronan. Biotechnol Adv 25: 537-557.

Sugahara KN, Murai T, Nishinakamura H, Kawashima H, Saya H, Miyasaka M (2003) Hyaluronan oligosaccharides induce CD44 cleavage and promote cell migration in CD44-expressing tumor cells. J Biol Chem 278: 32259-32265.

Tan J-X, Wang X-Y, Li H-Y, Su X-L, Wang L, Ran L, Zheng K, Ren G-S (2011) HYAL1 overexpression is correlated with the malignant behavior of human breast cancer. Int J Cancer 128: 1303-1315.

Taylor KR, Trowbridge JM, Rudisill JA, Termeer CC, Simon JC, Gallo RL (2004) Hyaluronan fragments stimulate endothelial recognition of injury through TLR4. J Biol Chem 279: 17079-17084.

Termeer C, Benedix F, Sleeman J, Fieber C, Voith U, Ahrens T, Miyake K, Freudenberg M, Galanos C, Simon JC (2002) Oligosaccharides of Hyaluronan activate dendritic cells via toll-like receptor 4. J Exp Med 195: 99-111.

Termeer CC, Hennies J, Voith U, Ahrens T, Weiss JM, Prehm P, Simon JC (2000) Oligosaccharides of hyaluronan are potent activators of dendritic cells. J Immunol 165: 1863-1870.

Tian X, Azpurua J, Hine C, Vaidya A, Myakishev-Rempel M, Ablaeva J, Mao Z, Nevo E, Gorbunova V, Seluanov A (2013) High-molecular-mass hyaluronan mediates the cancer resistance of the naked mole rat. Nature 499: 346-349.

Udabage L, Brownlee GR, Nilsson SK, Brown TJ (2005) The over-expression of HAS2, Hyal-2 and CD44 is implicated in the invasiveness of breast cancer. Exp Cell Res 310: 205-217.

Voelcker V, Gebhardt C, Averbeck M, Saalbach A, Wolf V, Weih F, Sleeman J, Anderegg U, Simon J (2008) Hyaluronan fragments induce cytokine and metalloprotease upregulation in human melanoma cells in part by signalling via TLR4. Exp Dermatol 17: 100-107.

West DC, Hampson IN, Arnold F, Kumar S (1985) Angiogenesis induced by degradation products of hyaluronic acid. Science 228: 1324-1326.

West DC, Kumar S (1989) The effect of hyaluronate and its oligosaccharides on endothelial cell proliferation and monolayer integrity. Exp Cell Res 183: 179-196.

Wiig H, Aukland K, Tenstad O (2003) Isolation of interstitial fluid from rat mammary tumors by a centrifugation method. Am J Physiol 284: H416-H424.

Yang C, Cao M, Liu H, He Y, Xu J, Du Y, Liu Y, Wang W, Cui L, Hu J, Gao F (2012) The high and low molecular weight forms of hyaluronan have distinct effects on CD44 clustering. J Biol Chem 287: 43094-43107.

Zhang L, Underhill CB, Chen L (1995) Hyaluronan on the surface of tumor cells is correlated with metastatic behavior. Cancer Res 55: 428-433.

This work is published under the standard license to publish agreement. After 12 months the work will become freely available and the license terms will switch to a Creative Commons AttributionNonCommercial-Share Alike 3.0 Unported License.

Supplementary Information accompanies this paper on British Journal of Cancer website (http://www.nature.com/bjc) 